\title{
1 Manipulation of metabolism in complex eukaryotic systems to \\ 2 control cellular state
}

3

4 Amy M. Su and Mark P. Styczynski*

5

6 School of Chemical \& Biomolecular Engineering, Georgia Institute of Technology, Atlanta, GA, 7 USA

8

9 *Corresponding author. Address: 311 Ferst Drive NW, Atlanta, GA 30332-0100, USA; email:

10 Mark.Styczynski@chbe.gatech.edu

11

12 Keywords:

13 Metabolism; stem cells; cancer; cancer stem cells; metabolites

14 


\section{Abstract}

16 The regulation of metabolism is critical to many important cellular processes in higher 17 eukaryotes, and metabolites themselves can have significant regulatory potential in complex 18 phenotypes. Thus, external control of metabolism for both biotechnological and biomedical ends 19 is of great importance. There has been increasing emphasis on using non-genetic approaches for 20 direct, transient control of metabolism and cellular state, with particularly promising applications 21 in stem cell biomanufacturing and cancer. Metabolite-based methods have been used to facilitate 22 expansion of stem cells, to control and prevent their differentiation, and to reprogram cells to a 23 pluripotent state. Similar approaches are also being explored to inhibit the growth of cancer cells. 
Metabolic control in complex eukaryotic systems has long been an important biotechnological goal. Cell culture engineering was key in enabling efficient, scalable production of antibodies and other proteins via Chinese hamster ovary $(\mathrm{CHO})$ cells grown in bioreactors $[1,2]$ as well other mammalian cell types [3], and improvement of these techniques is still an active research area [4]. For these applications, the endpoint is typically a comparatively simple phenotype: protein secretion. However, as biotechnological applications and the cell culture systems they employ become more diverse and complex, previously developed approaches to controlling mammalian cell metabolism will need to be repurposed and new methods to facilitate fine-tuned control of these systems will need to be developed.

One of the most promising applications of cell culture engineering is the emerging field of stem cell engineering and biomanufacturing [5,6]. Stem cells have significant potential as therapeutics for treating numerous diseases via both autologous and allogeneic approaches. Irrespective of the scientific and biomedical challenges that abound in the use of stem cells as therapeutics, there are at least two practical challenges in translating multipotent stem cells into a viable therapeutic platform: expansion to enable industrial-scale production of cells, and maintaining cells in, or differentiating them to, the appropriate cell type state. Moreover, if one considers the use of induced pluripotent stem cells (iPSCs) to enable autologous transplantation, efficient reprogramming of the cells is another key step that must be optimized. Ultimately, then, a much more complex phenotype must be accounted for in stem cells than in $\mathrm{CHO}$ cells: whereas $\mathrm{CHO}$ cells are the vehicle for making the desired product (i.e. protein), the stem cells themselves are the desired outcome of the bioprocess, and thus the cell type state (which has manifold possibilities) is the main target to be explicitly monitored and controlled. While some of the same principles used in $\mathrm{CHO}$ cell engineering can be applied, there is also increased demand for simple molecular mediators to help direct cells on the complex differentiation landscape, since genetic approaches are often to be avoided for therapeutic applications.

The principles for stem cell biomanufacturing can also be applied to another complex biological system with related, but often opposite, goals: cancer. Rather than encouraging cell growth as in

52 stem cell biomanufacturing, the goal is instead to stop proliferation of cancer cells (in some cases 
by inducing differentiation [7]). There are a surprising number of similarities between cancerous

54 cells and stem cells: not only can both cell types create many cells from a few (through proliferation or self-renewal), but they both rely on high glycolytic flux and have a number of uncommon metabolic tendencies [8]. In both cases, there is a preference to avoid genetic

57 interventions for manipulating the cells, whether due to difficulty in delivery, potential genetic side effects, or regulatory approval issues. Lastly, while small molecules have been dominant for decades in cancer treatment, the use of endogenous metabolites (or close derivatives or analogs) is beginning to show great potential, consistent with recent advances in using endogenous small molecules to control stem cells.

Here, we consider stem cells and cancer cells as two important challenges for controlling cell growth and cell state in complex systems. In both cases, metabolism has recently been shown to be critical in the cells' characteristic phenotypes, and metabolism-based control is emerging as a promising avenue to manipulate cell state and phenotype. We first address the prominent bioprocessing and biotechnological challenge of stem cell biomanufacturing. We then consider similarities between this field and the control of cancer metabolism, with brief discussion of the interesting intersection of these two cell types (cancer stem cells) and what this research area could mean for biomedical applications.

\section{Controlling stem cell state for biomanufacturing}

72 Stem cells are defined by their ability to self-renew (forming identical daughter cells) and differentiate (creating more specialized daughter cells), with many different types and classes of these cells. Embryonic stem cells (ESCs) are derived from preimplantation-stage embryos and are pluripotent, meaning they have the ability to generate any cell type, from any of the three germ layers. (They are not totipotent, though, which entails the additional ability to generate the extraembryonic cell types such as those in the placenta.) As cells differentiate, their range of possible lineages becomes more restricted (they lose potency, from pluripotent to multipotent to unipotent) until they differentiate to a terminal cell type. Multipotent cell types (that can form multiple terminal cell types) such as hematopoietic stem cells (HSCs) or mesenchymal stem cells (MSCs) can be derived from ESC lines or, more commonly, be collected from stem cell 
82 populations that persist in adults. Induced pluripotent stem cells (iPSCs) are created from 83 differentiated cells (often fibroblasts of various origin) that are reprogrammed back to a 84 pluripotent state by manipulation of the cells' gene regulatory network. iPSCs are particularly 85 exciting for not only their utility as a source of pluripotent stem cells, but also for the possibility 86 of personalized medicine at a previously unforeseen level of specificity. All of these cell types 87 represent complex phenotypes and cellular states that can be difficult to thoroughly characterize and demonstrate; often the expression of just a few known markers is used as a proxy for differentiation status, though in some cases more detailed functional characterization of cells is also performed.

One of the key practical barriers to stem cell-based therapeutics is the production of enough cells of the correct types for therapeutic doses at a commercial scale. With disease-dependent estimates ranging up to $10^{9}$ or more cells for a single dose [9], multiplied by thousands to millions of patients, a basic two-dimensional adherent culture approach clearly will not scale industrially. Three-dimensional bioreactor formats for culture and differentiation of pluripotent stem cells have sought to eliminate the dependence on tissue culture surface area (an excellent review is available elsewhere [9]). However, the dramatically different physical conditions of bioreactors as compared to adherent culture (introducing elements such as shear stress, degree of mixing, and gas phase composition) lend themselves to numerous challenges in meeting the metabolic needs of the cells. For example, higher cell density leads to greater consumption of nutrients and oxygen, which can be addressed via a well-mixed nutrient and oxygen supply throughout the tank and inside cellular aggregates, and via continuous reactor-level control of these parameters. Higher cell density also leads to greater production of $\mathrm{CO}_{2}$ that can stay dissolved in the bioreactor and tends to inhibit growth, which must be addressed. Work is ongoing in bioreactor development; one of the most promising recent approaches used a thermoresponsive hydrogel in fully defined conditions for long-term, serial expansion at a high rate (20-fold within 5 days) and for in-reactor differentiation [10]. As limitations on cell growth and culture are alleviated, even more attention will turn to the establishment of methods for precise control of cell type, particularly because changing culture formats will affect the signals that control differentiation, including endogenous factors such as extracellular matrix and cellsecreted growth factors. Metabolism-based control is a promising candidate to address this issue. 
113 There is significant evidence linking the metabolic state of pluripotent stem cells to their 114 differentiation state, suggesting the potential importance of metabolism in differentiation and 115 self-renewal. For example, via a metabolite profiling approach, the oxidation state of lipid 116 profiles was shown to be highly correlated with cell state during embryonic stem cell 117 differentiation [11]. Supplementation with metabolites associated with oxidative metabolism 118 promoted differentiation down neural and cardiac lineages as assayed by protein markers such as $119 \quad$ BIII-tubulin (neural) or cardiotroponin (cardiac), suggesting that the metabolic changes actually 120 played a functional role. Similar metabolite-focused investigations of induced pluripotent stem 121 cells also showed metabolic changes between induced pluripotent stem cells and their parental 122 cells [12,13] and differences between induced pluripotent and embryonic stem cells. Most 123 recently, it was found that metabolic changes are present even at extremely early stages of 124 differentiation [14], leading to identification of glycolysis-mediated changes in histone acetylation and metabolite levels that play a role in differentiation. Taken together, these results 126 suggest a central role of metabolism in differentiation and motivate the desire to control 127 metabolism in stem cells.

\section{Endogenous metabolites as soluble differentiation factors}

129 Supporting the hypothesis that metabolism plays a central role in differentiation is that most of 130 the above studies also showed that exogenous supplementation of metabolites could mediate 131 significant changes in differentiation efficiency. For example, in addition to the pro-cardiac and 132 pro-neural roles of certain lipids [11], acetate supplementation blocked loss of the pluripotency 133 marker Oct4 for at least four days in both human and mouse embryonic stem cells [14]. This is 134 suggestive of a broader link from metabolite levels to signaling pathways [15] and epigenetic 135 modifications (including histone acetylation and others) [16], consistent with the metabolic 136 changes found at early stages of differentiation.

137 The potential for metabolite-mediated control of differentiation is consistent with long138 established knowledge in the field, though recent advances in this area have been significant. For 139 example, retinoic acid has long been known to facilitate differentiation towards a neural fate [17] 140 via its role as a signaling molecule. L-proline has also been known to induce differentiation 
141 toward early primitive ectoderm-like cells, shown by maintenance of Oct4 and upregulation of 142 Otx2 and Dnmt3b [18]. Recently it was identified that L-proline can also induce a mesenchymal143 like invasive state in embryonic cells, with H3K9 and H3K36 histone methylation induced by 144 proline playing a significant role in the process [19]. In this case, the mesenchymal-like state 145 could be reversed by the addition of another metabolite, ascorbic acid. L-proline was also demonstrated to be involved in a feedback loop that modulates the Gcn2-Eif2 $\alpha$-Atf4 amino acid starvation response pathway and controls proliferation and the above-noted mesenchymal-like 148 state [20].

Numerous other similarly interesting discoveries have been made recently. In studying the metabolic similarities and differences between a naïve and a more committed ES cell state, it was found that cell-permeable $\alpha$-ketoglutarate could help maintain pluripotency and promote selfrenewal through its impact on $\alpha$-ketoglutarate-dependent demethylases acting on histone methylation, while cell-permeable succinate promoted differentiation [21]. Methionine metabolism was found to be critical in maintaining human pluripotent cells, with methionine deprivation prompting differentiation and prolonged deprivation leading to apoptosis. This was mediated through the conversion of methionine to S-adenosylmethionine (SAM), a crucial methyl donor for gene regulation [22]. Two antioxidants (N-acetyl-L-cysteine and ascorbic acid2-phosphate) significantly reduced doubling times and increased cell density in mesenchymal stem cells, mediated by regulation of CDK and its inhibitors [23]. Another interesting finding in a related vein is that culture on lactate in glucose-deprived conditions enabled purification of stem cell-derived cardiomyocytes [24]. While lactate did not directly promote differentiation towards a cardiomyocyte fate, the metabolic status and capabilities of different cell types were harnessed to improve stem cell engineering.

\section{Cell culture engineering approaches to affect metabolism and control cell state}

More traditional methods for controlling cell metabolism, such as control of oxygen levels and feeding regimes, have also been exploited to manipulate stem cell differentiation state (a detailed review was recently published [25]). For example, hematopoietic stem cells reside in a hypoxic niche in vivo, suggesting that low-oxygen culture may be appropriate [26]. In a perfused bioreactor model, careful control of $\mathrm{pO}_{2}$ at $30 \%$ air saturation was shown to improve human 
170

171

172

173

174

175

176

177

178

179

180

181

182

183

184

185

186

187

188

189

190

191

192

193

194

195

196

197

198

embryonic stem cell growth [27], though the same phenomenon was not observed for human mesenchymal stem cells [28]. In the latter case, not only did oxygen levels not affect growth, neither did three different feeding regimes (though slight metabolic differences were observed). More generally, recent evidence suggests that perfusion (versus batch culture) is particularly important to decrease metabolic stress and maintain cells in a pluripotent, easily expanded state [29].

\section{Reprogramming cell type mediated by metabolism and metabolites}

Not only have metabolic differences between induced pluripotent stem cells, their parental cells, and embryonic stem cells been identified $[12,13]$, but the functional roles of those differences have also been explored. For example, based on the metabolic changes in induced pluripotent stem cells, fructose-6-phosphate supplementation was found to significantly improve reprogramming efficiency, likely via stimulation of glycolysis [13]. Such an endogenous metabolite-based approach is consistent with general trends in induced pluripotent reprogramming techniques: moving away from transcription factor-based induction [30] to use more small molecules instead (including fructose-6-phosphate and fructose-2,6-bisphosphate) [31], and finally using only small molecules [32]. During this evolution of techniques, hypoxia was identified as improving reprogramming [33]. Additional metabolic mediators of reprogramming were also identified, including ascorbic acid's ability to increase reprogramming efficiency by alleviating cell senescence of the starting fibroblast culture [34]. Most recently, nicotinamide was used to protect cells from oxidative stress through replenishment of $\mathrm{NAD}^{+}$, which in turn helped avoid senescence and apoptosis, ultimately leading to increased reprogramming efficiency [35]. Reprogramming efficiency was evaluated by alkaline phosphatase staining, histological staining, protein markers of pluripotency (including SSEA3, SSEA4, and Nanog), teratoma formation in vivo, and the successful creation of chimeric mice.

\section{Manipulating metabolism for cancer therapies}

Another area where researchers are actively exploiting the manipulation of metabolism for control of cell state is cancer. Both cancer cells and stem cells are characterized by high glycolytic flux and the ability to divide rapidly, as well as other unique metabolic characteristics. 
It is thus reasonable that approaches used for controlling metabolism and cell state in stem cell culture could also be used to address cancerous proliferation. (A thorough primer of the basic biology and biochemistry of cancer metabolism is available [36], though without the latest findings in this rapidly-moving field.) The intersections between metabolism and signaling continue to be fleshed out; for example, the relationship between the oncogenic transcription factor c-MYC and proline metabolism was recently established [37]. (Note the overlap with proline's suggested role in stem cell differentiation.) Metabolism is thus considered a strong candidate target for anticancer therapies, ranging from reconsideration of long-standing approaches (e.g., targeting polyamine metabolism [38]) to exciting new targeted approaches. One prominent example is recent work targeting mutants of isocitrate dehydrogenase enzymes IDH1 and IDH2 (newly appreciated metabolic oncogenes), with significant promise for, among other applications, fighting leukemia [7,39]. A more exhaustive review of the potential for metabolism in cancer therapeutics is available elsewhere [40].

\section{Endogenous metabolites and antimetabolites to control cancer}

As in stem cell engineering, exploiting the effects of endogenous metabolites may hold significant promise for controlling metabolism and targeting cancer (Figure 2). The use of metabolite-like molecules is certainly not a new concept in the field: antimetabolites (inhibitors that closely mimic and outcompete endogenous metabolites) have been used for decades, including some of the earliest leukemia chemotherapeutics [41]. The use of antimetabolites is broadening, with clinical trials and preclinical studies underway [42].

More directly, though, metabolites themselves are being pursued for potential chemotherapeutic properties, with the thought that treating with endogenous metabolites could simulate a more normal cell state and trigger apoptosis [43]. For example, it has been known for decades that Vitamin K2 (menaquinone) has the potential for anticancer activity. A recent paper characterized the specific mechanism of menaquinone's action in a leukemia cell line through targeting Bak, which leads to mitochondrial-mediated apoptosis [44]. Other work showed that menaquinone can have therapeutic effects on prostate cancer cells, whether or not they were hormone-dependent, via caspase-3 and -8 dependent apoptosis [45]. Interestingly, recent metabolite profiling-driven work identified an unknown connection between menaquinone and phosphoethanolamine in the 
228 induction of apoptosis in a leukemic cell line [46]. This is particularly relevant given a recent 229 series of papers showing the anti-cancer effects of phosphoethanolamine on breast cancer, 230 leukemia, and renal cell carcinoma [47-49]. Metabolite-based therapeutics are particularly 231 exciting because, since they are already part of human metabolism and are likely well-tolerated, they could lead to the development of chemotherapeutics with fewer side effects.

\section{Metabolism in cancer stem cells}

An interesting research frontier at the intersection of cancer biology and stem cell biology is the study of cancer stem cells. These are a small subpopulation of chemoresistant, radioresistant cells capable of recapitulating an entire tumor. These cells are often left behind after initial cancer treatments and are thought to be a significant cause of cancer relapse. While the origin of these cells is debated, there is increasing acceptance of their potential importance, and growing interest in their metabolism as a possible source for insights into how to control or target them.

While cancer stem cell metabolism had been until recently all but ignored except for one paper characterizing the impacts of culturing glioma-initiating cells [50], in the past year there have been four papers studying cancer stem cell metabolism. Metabolite and proteome profiling on spheroid-cultured breast cancer stem cells helped identify their dependence on fermentative glycolysis, consistent with general expectations for cancer cells but different from the behavior observed for spheroid-derived adherent cells [51]. Cancer stem cells from a human colon adenocarcinoma cell line were isolated and their metabolite profiles measured in vitro, as well as impacts on the serum metabolome in an in vivo mouse model [52]. Finally, two papers helped to flesh out not only the baseline metabolic differences between an ovarian cancer stem cell line and its isogenic parental cells [53], but also the fundamentally different ways that each cell type's metabolism responds to environmental conditions likely present in the tumor microenvironment [54]. Thus, it seems that metabolic changes in cancer stem cells are not just due to different proliferation rates, but fundamentally different metabolic programs that could someday be used as the basis for therapeutics or adjuvants that specifically target cancer stem cell metabolism. 


\section{Conclusions}

257 In summary, it has been shown that metabolism has a great impact on complex eukaryotic 258 systems with biotechnological (stem cell biomanufacturing) and biomedical (stem cell-based regenerative medicine and cancer treatment) relevance. Thus, controlling metabolism in these systems is critical for achieving the desired biotechnological and biomedical ends. In both cases, we have seen that the levels of metabolites not only correlate with cell state, but can also directly affect cell state upon growth medium supplementation. We believe this is a promising future direction for the development of methods to control and manipulate both metabolism and cell state. However, to achieve this potential more research is needed into mapping metabolic states of complex systems and how these tie in to complex biological processes. A critical component of fine-tuned metabolic control is a greater understanding of system-level dynamics. One key approach which should see broader application is temporal metabolite profiling via metabolomics, which provides insight into the changing levels of a wide variety of metabolites simultaneously. This important tool continues to bolster our knowledge of cancer metabolism, revealing novel correlations which are not always apparent from more narrow metabolic studies; it is increasingly showing its value in application to the complex question of stem cell behavior and fate as well. In both of these cases, insights learned from metabolite profiling may provide leads on candidate small molecule mediators of cell behavior (whether proliferation or differentiation), or potential targets for such interventions. Metabolic flux analysis, which uses stable isotopic tracers to elucidate the distribution of metabolic pathway utilization, is another technique that should see broader application for better characterization of cancer and stem cells. Metabolic flux analysis pairs well with dynamic metabolomics measurements by providing more detailed and mechanistic (though typically more narrowly focused) insight, which will ultimately provide a deeper understanding of metabolic behavior in these cells and more chances to exert control over them. Additionally, the development of computational models incorporating data from these approaches and engineering tools to facilitate precise control of cellular state via metabolic pathways are critical for the field to continue to move forward.

\section{Acknowledgements}


The authors acknowledge support from the National Science Foundation (\#1125684) and the Semiconductor Research Corporation (task \#2484.001 in the Semiconductor Synthetic Biology program). The authors also thank Megan Cole for a critical review of the manuscript.

\section{References (annotations below)}

1. Borys MC, Linzer DI, Papoutsakis ET: Ammonia affects the glycosylation patterns of recombinant mouse placental lactogen-I by chinese hamster ovary cells in a $\mathrm{pH}$-dependent manner. Biotechnol Bioeng 1994, 43:505-514.

2. Xie L, Wang DI: High cell density and high monoclonal antibody production through medium design and rational control in a bioreactor. Biotechnol Bioeng 1996, 51:725-729.

3. Zhou W, Rehm J, Europa A, Hu WS: Alteration of mammalian cell metabolism by dynamic nutrient feeding. Cytotechnology 1997, 24:99-108.

4. Bradley SA, Ouyang A, Purdie J, Smitka TA, Wang T, Kaerner A: Fermentanomics: monitoring mammalian cell cultures with NMR spectroscopy. J Am Chem Soc 2010, 132:9531-9533.

5. Loring JF, McDevitt TC, Palecek SP, Schaffer DV, Zandstra PW, Nerem RM: A global assessment of stem cell engineering. Tissue Eng Part A 2014, 20:2575-2589.

6. Silva M, Daheron L, Hurley H, Bure K, Barker R, Carr AJ, Williams D, Kim HW, French A, Coffey PJ, et al.: Generating iPSCs: translating cell reprogramming science into scalable and robust biomanufacturing strategies. Cell Stem Cell 2015, 16:13-17.

7. Wang F, Travins J, DeLaBarre B, Penard-Lacronique V, Schalm S, Hansen E, Straley K, Kernytsky A, Liu W, Gliser C, et al.: Targeted inhibition of mutant IDH2 in leukemia cells induces cellular differentiation. Science 2013, 340:622-626.

8. Pereira SL, Rodrigues AS, Sousa MI, Correia M, Perestrelo T, Ramalho-Santos J: From gametogenesis and stem cells to cancer: common metabolic themes. Hum Reprod Update 2014, 20:924-943.

9. Serra M, Brito C, Correia C, Alves PM: Process engineering of human pluripotent stem cells for clinical application. Trends Biotechnol 2012, 30:350-359.

10. Lei $Y$, Schaffer DV: A fully defined and scalable 3D culture system for human pluripotent stem cell expansion and differentiation. Proc Natl Acad Sci U S A 2013, 110:E5039-5048.

11. Yanes O, Clark J, Wong DM, Patti GJ, Sanchez-Ruiz A, Benton HP, Trauger SA, Desponts C, Ding S, Siuzdak G: Metabolic oxidation regulates embryonic stem cell differentiation. Nat Chem Biol 2010, 6:411-417.

12. Meissen JK, Yuen BT, Kind T, Riggs JW, Barupal DK, Knoepfler PS, Fiehn O: Induced pluripotent stem cells show metabolomic differences to embryonic stem cells in polyunsaturated phosphatidylcholines and primary metabolism. PLoS One 2012, 7:e46770.

13. Panopoulos AD, Yanes O, Ruiz S, Kida YS, Diep D, Tautenhahn R, Herrerias A, Batchelder EM, Plongthongkum $\mathrm{N}$, Lutz $\mathrm{M}$, et al.: The metabolome of induced pluripotent stem cells reveals metabolic changes occurring in somatic cell reprogramming. Cell Res 2012, 22:168-177.

14. Moussaieff A, Rouleau M, Kitsberg D, Cohen M, Levy G, Barasch D, Nemirovski A, Shen-Orr S, Laevsky I, Amit $\mathrm{M}$, et al.: Glycolysis-mediated changes in acetyl-CoA and histone acetylation control the early differentiation of embryonic stem cells. Cell Metab 2015, 21:392-402.

15. Agathocleous M, Harris WA: Metabolism in physiological cell proliferation and differentiation. Trends Cell Biol 2013, 23:484-492. 
16. Moussaieff A, Kogan NM, Aberdam D: Energy Metabolites: Key Mediators of the Epigenetic State of Pluripotency. Stem Cells 2015.

17. Bain G, Kitchens D, Yao M, Huettner JE, Gottlieb DI: Embryonic stem cells express neuronal properties in vitro. Dev Biol 1995, 168:342-357.

18. Washington JM, Rathjen J, Felquer F, Lonic A, Bettess MD, Hamra N, Semendric L, Tan BS, Lake JA, Keough RA, et al.: L-Proline induces differentiation of ES cells: a novel role for an amino acid in the regulation of pluripotent cells in culture. Am J Physiol Cell Physiol 2010, 298:C982-992.

19. Comes S, Gagliardi M, Laprano N, Fico A, Cimmino A, Palamidessi A, De Cesare D, De Falco S, Angelini C, Scita G, et al.: L-Proline Induces a Mesenchymal-like Invasive Program in Embryonic Stem Cells by Remodeling H3K9 and H3K36 Methylation. Stem Cell Reports 2013, 1:307-321.

20. D'Aniello C, Fico A, Casalino L, Guardiola O, Di Napoli G, Cermola F, De Cesare D, Tate R, Cobellis G, Patriarca EJ, et al.: A novel autoregulatory loop between the Gcn2-Atf4 pathway and L-Proline metabolism controls stem cell identity. Cell Death Differ 2015, 22:1094-1105.

21. Carey BW, Finley LW, Cross JR, Allis CD, Thompson CB: Intracellular alpha-ketoglutarate maintains the pluripotency of embryonic stem cells. Nature 2015, 518:413-416.

22. Shiraki N, Shiraki Y, Tsuyama T, Obata F, Miura M, Nagae G, Aburatani H, Kume K, Endo F, Kume S: Methionine metabolism regulates maintenance and differentiation of human pluripotent stem cells. Cell Metab 2014, 19:780-794.

23. Sun LY, Pang CY, Li DK, Liao CH, Huang WC, Wu CC, Chou YY, Li WW, Chen SY, Liu HW, et al.: Antioxidants cause rapid expansion of human adipose-derived mesenchymal stem cells via CDK and CDK inhibitor regulation. J Biomed Sci 2013, 20:53.

24. Tohyama S, Hattori F, Sano M, Hishiki T, Nagahata Y, Matsuura T, Hashimoto H, Suzuki T, Yamashita $\mathrm{H}$, Satoh $\mathrm{Y}$, et al.: Distinct metabolic flow enables large-scale purification of mouse and human pluripotent stem cell-derived cardiomyocytes. Cell Stem Cell 2013, 12:127-137.

25. Sart S, Agathos SN, Li Y: Process engineering of stem cell metabolism for large scale expansion and differentiation in bioreactors. Biochemical Engineering Journal 2014, 84:74-82.

26. Simsek T, Kocabas F, Zheng J, Deberardinis RJ, Mahmoud AI, Olson EN, Schneider JW, Zhang CC, Sadek HA: The distinct metabolic profile of hematopoietic stem cells reflects their location in a hypoxic niche. Cell Stem Cell 2010, 7:380-390.

27. Serra M, Brito C, Sousa MF, Jensen J, Tostoes R, Clemente J, Strehl R, Hyllner J, Carrondo MJ, Alves PM: Improving expansion of pluripotent human embryonic stem cells in perfused bioreactors through oxygen control. J Biotechnol 2010, 148:208-215.

28. Dos Santos F, Campbell A, Fernandes-Platzgummer A, Andrade PZ, Gimble JM, Wen Y, Boucher S, Vemuri MC, da Silva CL, Cabral JM: A xenogeneic-free bioreactor system for the clinical-scale expansion of human mesenchymal stem/stromal cells. Biotechnol Bioeng 2014, 111:11161127.

29. Yeo D, Kiparissides A, Cha JM, Aguilar-Gallardo C, Polak JM, Tsiridis E, Pistikopoulos EN, Mantalaris A: Improving embryonic stem cell expansion through the combination of perfusion and Bioprocess model design. PLoS One 2013, 8:e81728.

30. Takahashi K, Tanabe K, Ohnuki M, Narita M, Ichisaka T, Tomoda K, Yamanaka S: Induction of pluripotent stem cells from adult human fibroblasts by defined factors. Cell 2007, 131:861-872.

31. Zhu S, Li W, Zhou H, Wei W, Ambasudhan R, Lin T, Kim J, Zhang K, Ding S: Reprogramming of human primary somatic cells by OCT4 and chemical compounds. Cell Stem Cell 2010, 7:651-655.

32. Hou P, Li Y, Zhang X, Liu C, Guan J, Li H, Zhao T, Ye J, Yang W, Liu K, et al.: Pluripotent stem cells induced from mouse somatic cells by small-molecule compounds. Science 2013, 341:651-654.

33. Yoshida Y, Takahashi K, Okita K, Ichisaka T, Yamanaka S: Hypoxia enhances the generation of induced pluripotent stem cells. Cell Stem Cell 2009, 5:237-241. 
34. Esteban MA, Wang T, Qin B, Yang J, Qin D, Cai J, Li W, Weng Z, Chen J, Ni S, et al.: Vitamin C enhances the generation of mouse and human induced pluripotent stem cells. Cell Stem Cell 2010, 6:71-79.

35. Son MJ, Son MY, Seol B, Kim MJ, Yoo CH, Han MK, Cho YS: Nicotinamide overcomes pluripotency deficits and reprogramming barriers. Stem Cells 2013, 31:1121-1135.

36. Vander Heiden MG, Cantley LC, Thompson CB: Understanding the Warburg effect: the metabolic requirements of cell proliferation. Science 2009, 324:1029-1033.

37. Liu W, Le A, Hancock C, Lane AN, Dang CV, Fan TW, Phang JM: Reprogramming of proline and glutamine metabolism contributes to the proliferative and metabolic responses regulated by oncogenic transcription factor c-MYC. Proc Natl Acad Sci U S A 2012, 109:8983-8988.

38. Battaglia V, DeStefano Shields C, Murray-Stewart T, Casero RA, Jr.: Polyamine catabolism in carcinogenesis: potential targets for chemotherapy and chemoprevention. Amino Acids 2014, 46:511-519.

39. Rohle D, Popovici-Muller J, Palaskas N, Turcan S, Grommes C, Campos C, Tsoi J, Clark O, Oldrini B, Komisopoulou E, et al.: An inhibitor of mutant IDH1 delays growth and promotes differentiation of glioma cells. Science 2013, 340:626-630.

40. Vander Heiden MG: Targeting cancer metabolism: a therapeutic window opens. Nature Reviews Drug Discovery 2011, 10:671-684.

41. Bobrovnikova-Marjon E, Hurov JB: Targeting metabolic changes in cancer: novel therapeutic approaches. Annu Rev Med 2014, 65:157-170.

42. Amelio I, Cutruzzola F, Antonov A, Agostini M, Melino G: Serine and glycine metabolism in cancer. Trends Biochem Sci 2014, 39:191-198.

43. Arakaki AK, Mezencev R, Bowen NJ, Huang Y, McDonald JF, Skolnick J: Identification of metabolites with anticancer properties by computational metabolomics. Mol Cancer 2008, 7:57.

44. Karasawa S, Azuma M, Kasama T, Sakamoto S, Kabe Y, Imai T, Yamaguchi Y, Miyazawa K, Handa H: Vitamin K2 covalently binds to Bak and induces Bak-mediated apoptosis. Mol Pharmacol 2013, 83:613-620.

45. Samykutty A, Shetty AV, Dakshinamoorthy G, Kalyanasundaram R, Zheng G, Chen A, Bosland MC, Kajdacsy-Balla A, Gnanasekar M: Vitamin k2, a naturally occurring menaquinone, exerts therapeutic effects on both hormone-dependent and hormone-independent prostate cancer cells. Evid Based Complement Alternat Med 2013, 2013:287358.

46. Dhakshinamoorthy S, Dinh N, Skolnick J, Styczynski MP: Metabolomics identifies the intersection of phosphoethanolamine with menaquinone-triggered apoptosis in an in vitro model of leukemia. Molecular Biosystems 2015, Advance Article.

47. Ferreira AK, Freitas VM, Levy D, Ruiz JL, Bydlowski SP, Rici RE, Filho OM, Chierice GO, Maria DA: Antiangiogenic and anti-metastatic activity of synthetic phosphoethanolamine. PLOS One 2013, 8:e57937.

48. Ferreira AK, Meneguelo R, Pereira A, Filho OM, Chierice GO, Maria DA: Synthetic phosphoethanolamine induces cell cycle arrest and apoptosis in human breast cancer MCF-7 cells through the mitochondrial pathway. Biomed Pharmacother 2013, 67:481-487.

49. Ferreira AK, Santana-Lemos BA, Rego EM, Filho OM, Chierice GO, Maria DA: Synthetic phosphoethanolamine has in vitro and in vivo anti-leukemia effects. Br J Cancer 2013, 109:2819-2828.

50. Mlynarik V, Cudalbu C, Clement V, Marino D, Radovanovic I, Gruetter R: In vivo metabolic profiling of glioma-initiating cells using proton magnetic resonance spectroscopy at 14.1 Tesla. NMR Biomed 2012, 25:506-513. 
51. Ciavardelli D, Rossi C, Barcaroli D, Volpe S, Consalvo A, Zucchelli M, De Cola A, Scavo E, Carollo R, D'Agostino $D$, et al.: Breast cancer stem cells rely on fermentative glycolysis and are sensitive to 2-deoxyglucose treatment. Cell Death Dis 2014, 5:e1336.

52. Vincent Z, Urakami K, Maruyama K, Yamaguchi K, Kusuhara M: CD133-positive cancer stem cells from Colo205 human colon adenocarcinoma cell line show resistance to chemotherapy and display a specific metabolomic profile. Genes Cancer 2014, 5:250-260.

53. Vermeersch KA, Wang L, Mezencev R, McDonald JF, Styczynski MP: OVCAR-3 Spheroid-Derived Cells Display Distinct Metabolic Profiles. PLoS One 2015, 10:e0118262. cancer stem cell line. BMC Syst Biol 2014, 8:134. 


\section{Reference Annotations}

431 [7] ** This work showed that selectively inhibiting mutated forms of the enzyme IDH2 in

432 leukemia cells could induce differentiation. This work supports the scientific basis of a 433 chemotherapeutic currently in clinical trials and showing great potential.

$434[10] * *$ The authors developed a thermoresponsive hydrogel-based 3D culture system that allows 435 for completely defined, xeno-free conditions and easy passaging by temperature-controlled phase 436 change of the gel. This work demonstrated efficient and rapid expansion of pluripotent stem 437 cells.

438 [14]* Metabolomics was used to identify extremely early changes in metabolite profiles during 439 differentiation, and helped link metabolism to differentiation and histone acetylation. The 440 authors also found that acetate supplementation can delay differentiation.

441 [21] ** The metabolic similarities and differences between a naïve and a more committed ES 442 cell state were characterized, with the results leading to the discovery that the $\alpha$-ketoglutarate to 443 succinate ratio in mouse ESCs plays a key role in maintaining pluripotency, likely through 444 demethylation of repressive chromatin marks. This provides more evidence that supplementation 445 of endogenous metabolites can manipulate stem cell metabolism and differentiation state.

446 [24] * By withdrawing glucose and providing only lactate, pluripotent stem cell-derived 447 cardiomyocytes can be selected for based on their unique metabolic characteristics. This work 448 enabled significant improvement in differentiated culture purity with little manipulation of cells.

449 [35] ** This work demonstrated that nicotinamide can enhance reprogramming efficiency for 450 human induced pluripotent stem cells, through a variety of suspected mechanisms. It revealed the 451 importance of $\mathrm{NAD}^{+}$metabolism on pluripotency.

452 [46] * The authors characterized the metabolic impact of using an endogenous metabolite 453 (menaquinone) as an anti-cancer agent. Via metabolomics data, they identified a seemingly 454 unrelated metabolite from a different pathway as being involved in the activity of menaquinone 455 and as having anti-cancer activity itself. 
$456[54] *$ The authors found that metabolic responses to environmental perturbations varied between

457 cancer stem cells and isogenic parental cells. This was the first study to show a fundamentally 458 different dynamic metabolic program of cancer stem cells. 


\section{Figure Captions}

461 Figure 1. Metabolite-based control of stem cell state. A) Some of the main influences on

462 pluripotent stem cell differentiation and maintenance of pluripotency. Small molecules (like

463 metabolites) and culture conditions (like oxygen levels), which can be directly controlled, can

464 have a significant impact on cell state. Shear stress from any fluid flow in the culture and 465 mechanical forces like the stiffness of the cell culture surface can also affect cell state and fate.

466 Autocrine and paracrine factors produced by the cells also play a significant role in determining 467 cell state. B) A summary of the small molecule (metabolite) mediators of cell state described in 468 this review. Cell states including expansion and differentiation to cardiac and neural lineages are 469 represented, along with some metabolites that tend to promote these cell states. These molecules 470 cannot alone induce these transitions, but can play an important role in promoting or preventing 471 them. The bottom, dotted arrow represents the reprogramming pathway from fully differentiated 472 cells back to induced pluripotent stem cells, along with metabolites shown to aid in that process. 473 For all cases, the metabolites listed are not exhaustive, but are representative of recent work in 474 the field.

Figure 2. Metabolite links to cancer and anticancer activity. As normal cells (blue) are 477 transformed to cancerous cells (green), a number of metabolites are correlated with this process 478 and may even play an active role (with 2-hydroxyglutarate being perhaps the most well-known 479 oncometabolite). However, a number of metabolites or close derivatives thereof have also been 480 shown in vitro to have the potential for antiproliferative activity. The rare cancer stem cells 481 (yellow) in the tumor are often resistant to standard chemotherapeutics, but recent work suggests 482 that their metabolism could serve as a viable target to selectively treat them or push them away 483 from a cancer stem cell state. 
a)

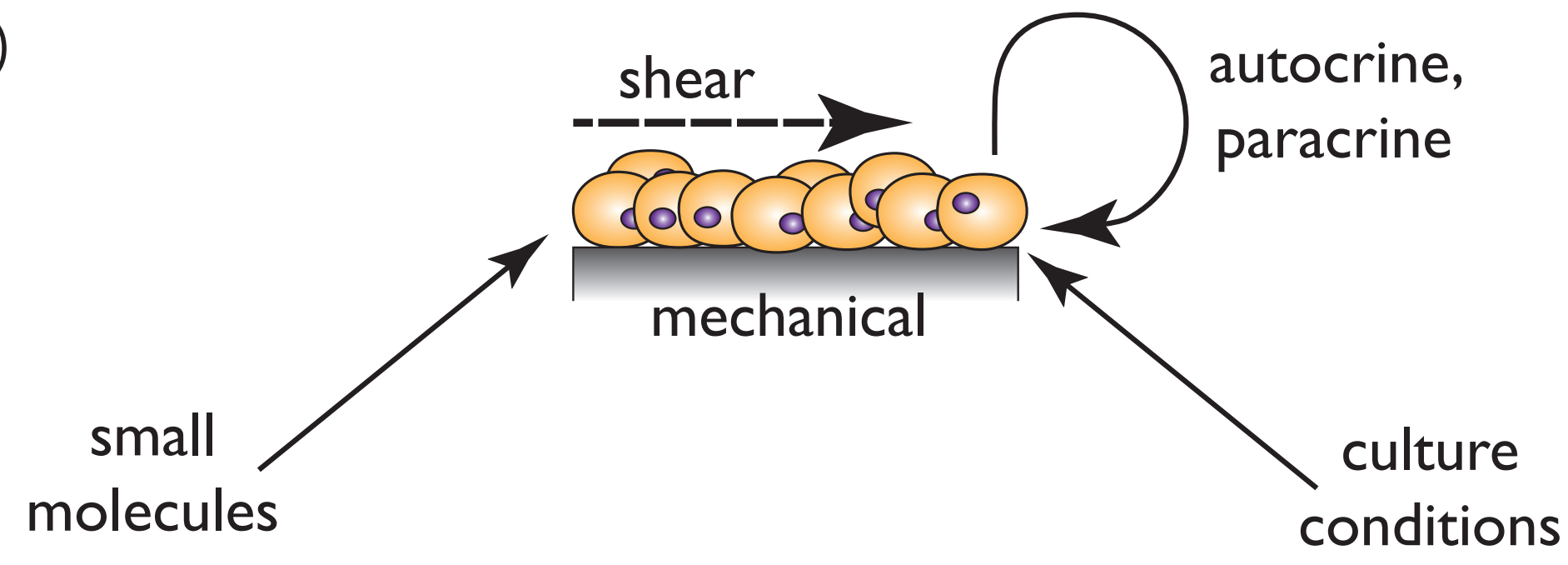

b)

ascorbic acid-2-phosphate, a-ketoglutarate, acetate
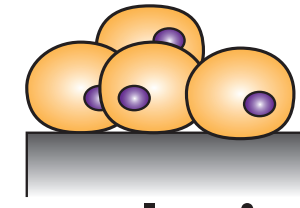
pluripotent

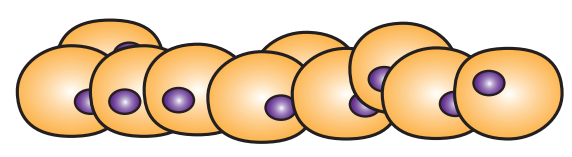

expansion
(pluripotent)

cardiac

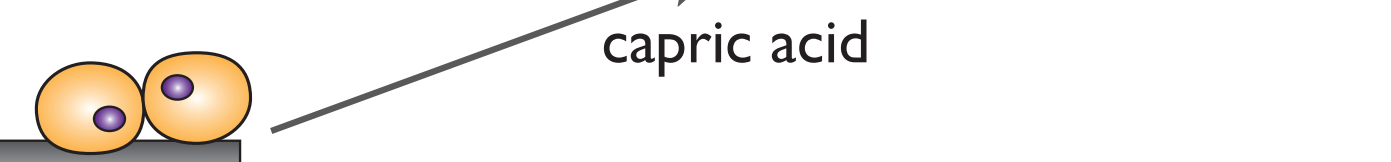

retinoic acid,

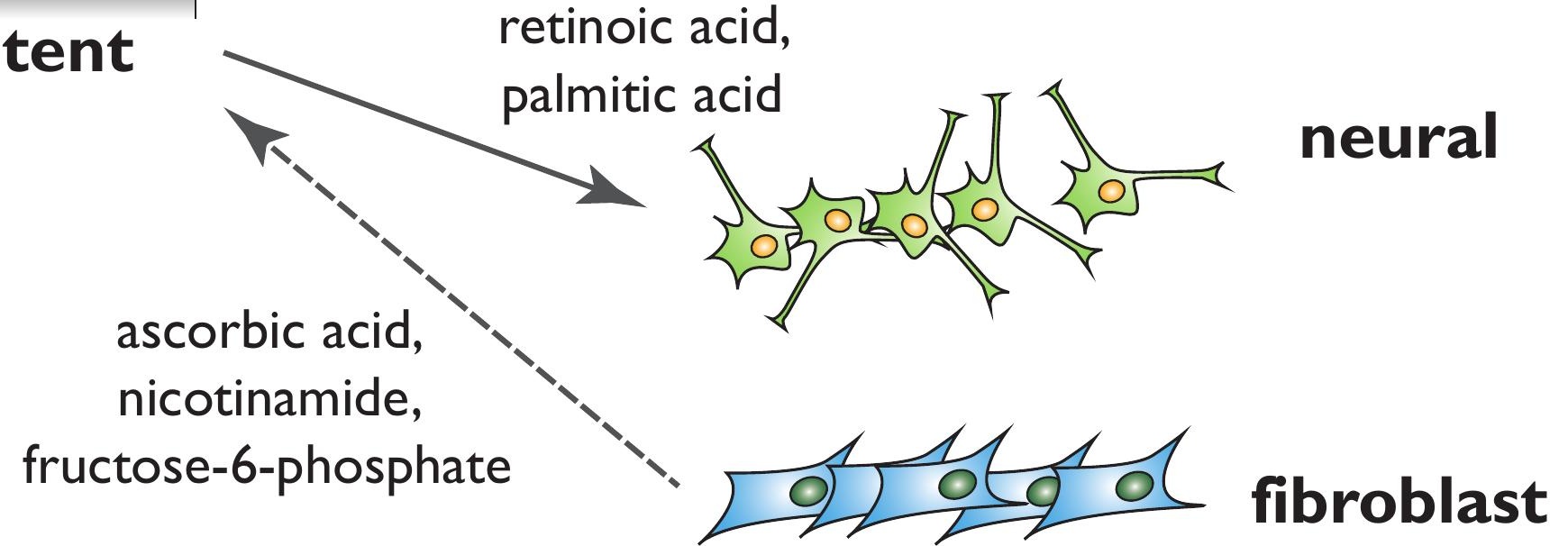


Figure 2

2-hydroxyglutarate, proline

\section{normal cells}

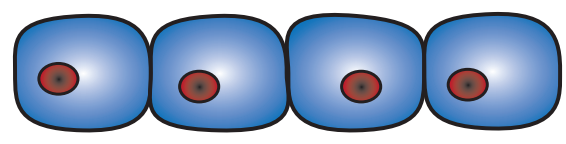

tumor cells
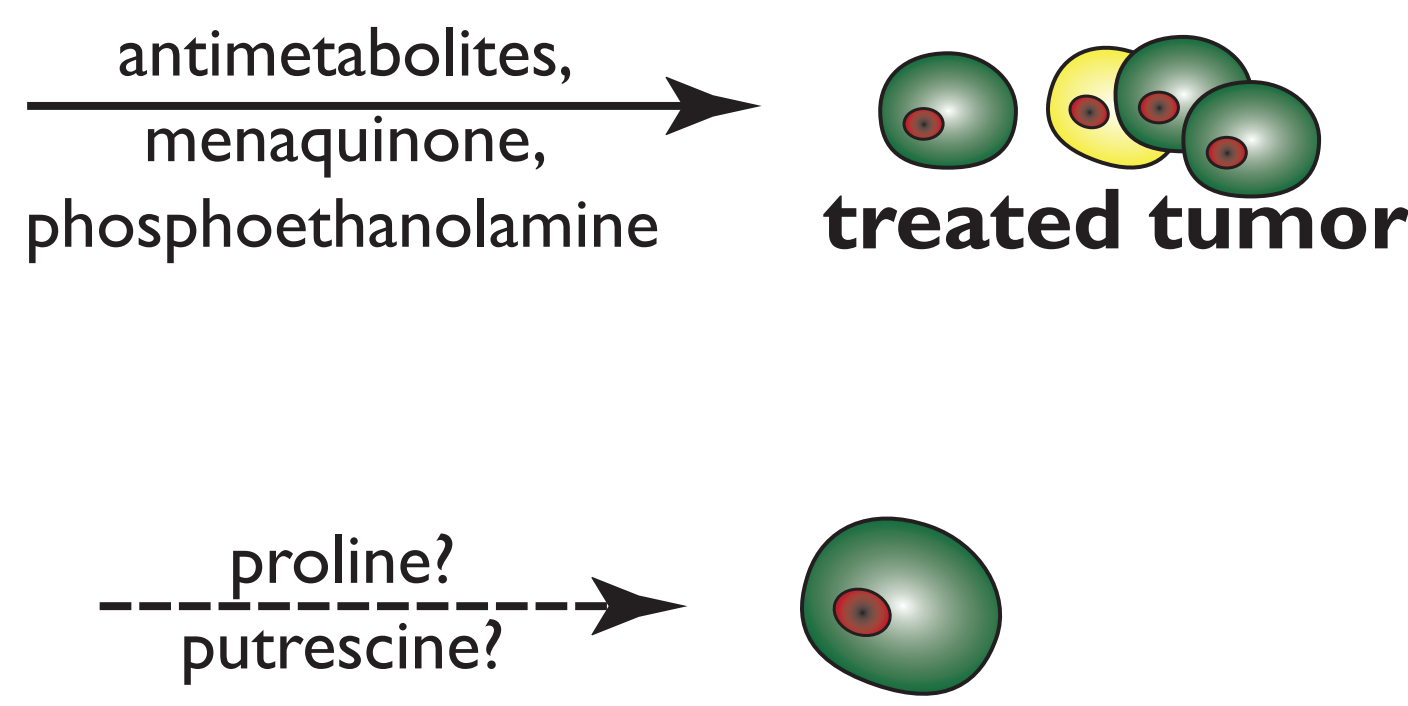

cancer stem cell 